\title{
An improved method for guinea pig airway smooth muscle cell culture and the effect of SPFF on intracellular calcium
}

\author{
JIAN LIU ${ }^{1}$, YUYANG ZHANG ${ }^{1}$, QIAN LI ${ }^{1}$, QUANKUN ZHUANG ${ }^{1}$, \\ XIAOJIE ZHU ${ }^{1}$, LI PAN ${ }^{2,3}$ and MAOSHENG CHENG ${ }^{2,3}$ \\ ${ }^{1}$ School of Life Science and Biopharmaceutics, Shenyang Pharmaceutical University; ${ }^{2}$ School of Pharmaceutical Engineering, \\ Shenyang Pharmaceutical University; ${ }^{3}$ Key Laboratory of Structure Based Drug Design and Discovery, \\ Ministry of Education, Shenyang, Liaoning 110016, P.R. China
}

Received October 2, 2013; Accepted November 8, 2013

DOI: $10.3892 / \mathrm{mmr} .2014 .2385$

\begin{abstract}
The aim of the present study was to establish an improved method for in vitro guinea pig airway smooth muscle (ASM) cell culture and to evaluate the effect of 2-(4-amino3-chloro-5-trifluomethyl-phenyl)-2-tert-butylamino-ethanol hydrochloride (SPFF), a novel $\beta 2$-adrenoceptor agonist, on the release of intracellular calcium in cells. A procedure for the efficient isolation, culture, passage and characterization of the cells was described. Primary ASM cells of guinea pigs were cultured by modified tissue cultivation. The cells were identified by their morphological characteristics and immunocytochemistry. The relative inhibition of the release of intracellular calcium by drugs in the cells was measured by fluorometric quantification with fluorochrome Fura-2/AM. The results were as follows: a) The ASM cells of the guinea pigs were successfully cultured and subcultured by using our improved method and typical peak-valley characteristics were observed under the phase contrast microscope; $b$ ) data from immunocytochemical staining with specific $\alpha$-smooth muscle actin ( $\alpha$-SMA) demonstrated that the cells were ASM cells; c) the growth characteristics and cell viability demonstrated that the cells were in good condition and were able to be applied in the follow-up studies; d) the inhibitory effect of SPFF on the release of intracellular calcium was concentration-dependent when compared with the control and e) the potential mechanisms of SPFF on the inhibition of intracellular calcium may
\end{abstract}

Correspondence to: Professor Yuyang Zhang, Department of Pharmacology, School of Life Science and Biopharmaceutics, Shenyang Pharmaceutical University, 103 Wenhua Road, Shenyang, Liaoning 110016, P.R. China

E-mail: 13614053862@163.com

Professor Maosheng Cheng, Department of Medicinal Chemistry, School of Pharmaceutical Engineering, Shenyang Pharmaceutical University, 103 Wenhua Road, Shenyang, Liaoning 110016, P.R. China E-mail: mscheng@263.net

Key words: guinea pigs, airway smooth muscle cells, identification, SPFF, intracellular calcium be independent of the ryanodine receptor, but may be closely associated with the inositol 1,4,5-trisphosphate receptor.

\section{Introduction}

Airway smooth muscle (ASM) cells are the main components of the respiratory tract system, which have important physiological roles, including maintaining the entire airway structure and keeping airway tension normal. In recent years, studies have indicated that cell function is closely correlated with the pathophysiological changes of respiratory diseases, including the proliferation of airway smooth muscle cells, airway inflammation and airway remodeling. ASM is important in almost all the pathophysiological and clinical aspects of respiratory illness, including bronchial asthma and chronic obstructive pulmonary disease (COPD) (1).

A better understanding of the regulatory factors in airways may be beneficial for elucidating the pathogenesis of respiratory illnesses. The function of ASM cells is regulated via intracellular signaling pathways, involving $\mathrm{Ca}^{2+}$ handling, enzymatic activity as well as protein phosphorylation. Among all the factors, intracellular $\mathrm{Ca}^{2+}$ may be crucial. An increase in the intracellular $\mathrm{Ca}^{2+}$ concentration is a key signal in initiating and maintaining a variety of cellular responses, including ASM contraction, cell proliferation and migration and gene expression in ASM cells (2). Studies have demonstrated that $\mathrm{Ca}^{2+}$, which was released from the sarcoplasmic reticulum (SR), was mainly regulated by the stimulated inositol 1,4,5-trisphosphate receptor $\left(\mathrm{IP}_{3} \mathrm{R}\right)$ and the ryanodine receptor $(\mathrm{RyR})(3,4)$. Stimulation of G protein-coupled receptors leads to the activation of phospholipase C (PLC), which then induces the production of the second messengers inositol 1,4,5-trisphosphate $\left(\mathrm{IP}_{3}\right)$ and diacylglycerol (DAG). When $\mathrm{IP}_{3}$ binds to its receptor $\mathrm{IP}_{3} \mathrm{R}$ located on the $\mathrm{SR}$, the released $\mathrm{Ca}^{2+}$ is able to activate $\mathrm{Ca}^{2+}$-calmodulin-dependent myosin light chain kinase, which finally leads to the contraction of respiratory tract smooth muscles (5). Furthermore, RyR is also involved in calcium channel modulation. It is able to generate self-sustaining intracellular calcium oscillations via a mechanism called calcium-induced calcium release (6).

2-(4-amino-3-chloro-5-trifluomethyl-phenyl)-2-tert-butyla mino-ethanol hydrochloride (SPFF), a novel $\beta 2$-adrenoceptor 
agonist, was synthesized and developed by the chemical synthetic laboratory of Shenyang Pharmaceutical University (Shenyang, Liaoning, China). The molecular structure of SPFF is displayed in Fig. 1. Previous studies demonstrated that SPFF was a potent, long-acting bronchodilator with relatively high $\beta 2$-adrenoceptor selectivity (7). The present study established a method for guinea pig airway smooth muscle cell culture in vitro and improved it in order to acquire a sufficient quantity of the cells. The effect of SPFF on the intracellular $\mathrm{Ca}^{2+}$ concentration and its potential mechanisms were investigated in the cultured ASM cells.

\section{Materials and methods}

Animals. Male or female hartley guinea pigs weighing 150-200 g were provided by the Experimental Animal Center of Shenyang Pharmaceutical University (Shenyang, Liaoning, China). Animals were bred in a facility controlled by temperature $\left(26 \pm 3^{\circ} \mathrm{C}\right)$, relative humidity $(50 \pm 5 \%)$ and light (14 and $10 \mathrm{~h}$ of light and dark), with free access to food and water.

Drugs and reagents. SPFF was supplied by the School of Pharmaceutical Engineering, Shenyang Pharmaceutical University (Shenyang, Liaoning, China; ee>99\%). Dulbecco's modified Eagle's medium (DMEM) and Hanks' balanced salt solution (HBSS) were purchased from Gibco-BRL (Carlsbad, CA, USA). Triton X-100 and MTT were obtained from Ameresco (Solon, OH, USA). 2-Aminoethyl diphenylborinate (2-APB) and dantrolene sodium salt were obtained from Sigma (St. Louis, MO, USA). Fura-2/AM was purchased from the Jiangsu Beyotime Institute of Biotechnology (Haimen, Jiangsu, China). Mouse anti- $\alpha$-smooth muscle actin ( $\alpha$-SMA), the streptavidin-biotin complex immunohistochemical staining kit and the diaminobenzidine staining kit were purchased from Wuhan Boster Biological Technology, Ltd. (Wuhan, China) and fetal bovine serum (FBS) was obtained from Tianjin Hualida Biotechnology Co., Ltd. (Tianjin, China). Mabuterol was provided by the School of Pharamceutical Engineering, Shenyang Pharmaceutical University (Shenyang, Liaoning, China).

\section{Method}

Guinea pig ASM cell culture. ASM bundles were isolated from guinea pig tracheas (five males and five females each time), which underwent surgical resection in accordance with procedures approved by the ethics committee of Shenyang Pharmaceutical University. Guinea pig ASM was cut into pieces of $1 \mathrm{~mm}^{3}$ using iridectomy scissors following washing in pre-cooled Hank's balanced salt solution (HBSS). The pieces were then transferred into a $100 \mathrm{~cm}^{2}$ cell culture flask coated preliminarily with FBS. The flask was agitated to allow the pieces to firmly attach to the flask wall and placed in a humidified incubator at $37^{\circ} \mathrm{C}$ and $5 \% \mathrm{CO}_{2}$. The flask was turned over following 16-20 h. DMEM containing $15 \% \mathrm{FBS}, 100 \mathrm{IU} / \mathrm{ml}$ penicillin, $100 \mathrm{IU} / \mathrm{ml}$ streptomycin and $2 \mathrm{mg} / \mathrm{ml} \mathrm{L-glutamine}$ were added to the flask, and the cells were further incubated. In accordance with the routine culture procedure, the medium was replaced every three days.

Once the cells migrated out of tissue blocks and grew to $90 \%$ confluence, they were passaged using $0.25 \%$ trypsin in
$0.02 \%$ EDTA. Cells at passage 3-10 were used in the subsequent experiments.

Identification of the guinea pig ASM cells. Cell morphology, including dimension and arrangement, was observed under an optical microscope (IX71; Olympus, Tokyo, Japan).

Immunocytochemical identification by $\alpha$-SMA was performed. In brief, cells at passages 5-8 were digested by $0.25 \%$ trypsin and $95 \%$ viability was identified by trypan blue exclusion. The cells were seeded in 24-well culture plates preliminarily coated with poly-L-lysine in a humidified incubator at $37^{\circ} \mathrm{C}$ and $5 \% \mathrm{CO}_{2}$ for $24 \mathrm{~h}$. The plates were rinsed three times using $0.01 \mathrm{~mol} / 1$ of phosphate-buffered saline and fixed in $4 \%$ phosphate-buffered paraformaldehyde for $30 \mathrm{~min}$ at room temperature. Following that, they were rinsed a further three times and treated with $0.25 \%$ Triton $\mathrm{X}-100$ for $10 \mathrm{~min}$ at room temperature. Then, they were blocked with $5 \%$ bovine serum albumin (Ameresco, Solon, OH, USA) for $30 \mathrm{~min}$ at room temperature following being rinsed three times. Then they were rinsed three times again and incubated with $\alpha$-SMA $(1: 200)$ at $4^{\circ} \mathrm{C}$ overnight. Following $12 \mathrm{~h}$, the plates were rinsed three times and treated according to the manufacturer's instructions of the SABC immunohistochemical staining kit and then those of the DAB staining kit. The results from the immunoreactive bands were determined according to the manufacturer's instructions and images were captured.

Identification of the growth characteristics of guinea pig ASM cells. The cells were seeded in 96-well culture plates following digestion with $0.25 \%$ trypsin. The viability was determined using an MTT assay as previously described (8) following day $1,2,3,4,5,6$ and 7, respectively. Cell viability and doubling time reflected the growth characteristics of guinea pig ASM cells.

Determination of intracellular calcium levels and their impairment by SPFF. The cells were digested by $0.25 \%$ trypsin when they grew to $90 \%$ confluence. Following being resuspended by fresh DMEM, they were seeded in black 96-well culture plates for $48 \mathrm{~h}$ in a humidified incubator at $37^{\circ} \mathrm{C}$ and $5 \% \mathrm{CO}_{2}$. The plates were rinsed twice with HBSS following the removal of DMEM. The cells were loaded with $5 \mu \mathrm{M}$ Fura-2/AM for $45 \mathrm{~min}$ on a microscale shaker at room temperature following treatment with SPFF or Mabuterol for $30 \mathrm{~min}$ at $37^{\circ} \mathrm{C}$. Then, the loading buffer was removed and cells were rinsed twice with HBSS to remove excess fluorescence dye. A suitable quantity of HBSS was added to maintain the cells in a basic physiological state. Calcium fluorescence intensity was determined by a multi-function microplate reader (3925; Corning America, Corning, NY, USA) at an excitation wavelength of $340 \mathrm{~nm}$ and an emission wavelength of $510 \mathrm{~nm}$. The relative calcium inhibition rate was determined using the following formula: (fluorescence intensity in the control group-fluorescence intensity in the tested group)/fluorescence intensity in the control group.

Investigation of the correlation between SPFF intervention, $I P_{3} R$ and $R y R$. The cells were treated with $2-\mathrm{APB}$, an $\mathrm{IP}_{3} \mathrm{R}$ blocker, and dantrolene, a RyR blocker, for $30 \mathrm{~min}$ at $37^{\circ} \mathrm{C}$ 


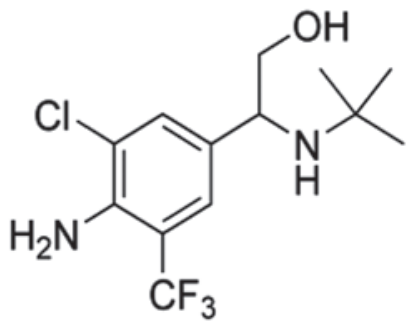

Figure 1. Molecular structure of SPFF. SPFF, 2-(4-amino-3-chloro-5trifluomethyl-phenyl)-2-tert-butylamino-ethanol hydrochloride.

following the addition of SPFF. Then the reaction system was loaded with $5 \mu \mathrm{M}$ Fura-2/AM for 45 min on a microscale shaker at room temperature. The calcium fluorescence intensity of the different treated groups was determined using a multi-function microplate reader. The potential inhibitive effect of SPFF on the release of intracellular calcium was analyzed according to changes in calcium fluorescence intensity.

Statistical analysis. Data are expressed as the mean \pm standard deviation. The significance between groups was determined using one-way analysis of variance with SPSS 16.0 software (SPSS Inc, Chicago, IL, USA). P $<0.05$ was considered to indicate a statistically significant difference.

\section{Results}

Primary guinea pig ASM cells. The cells migrated out from the tissue masses (indicated by a hollow arrowhead in Fig. 2A and B) grew and spread out in a radiating shape (indicated by short arrowheads in Fig. 2A) following 5-7 days of using the improved abovementioned method. The number of cells was significantly increased and fine confluence was able to be observed among adjacent cells 8-10 days later (indicated by short arrowheads in Fig. 2B). Cell confluence gradually became apparent 11-14 days later. Furthermore, interlaced reticulation around cells began to appear and then gradually demonstrated numerous branches when the density of the cells was highly increased (indicated by short arrowheads in Fig. 2C).

Guinea pig ASM cells following passage. The cells that grew up to $80 \%$ confluence gradually underwent morphological changes following treatment with $0.25 \%$ trypsin in $0.02 \%$ EDTA, including shrinking into a ball and becoming bright under the optical microscope, which indicated their cytoplasmic rebound (indicated by short arrowheads in Fig. 3A). Cells that were firstly subcultured and then partly transferred into a new cell culture flask attached on the lining surface of the culture flask $12 \mathrm{~h}$ later (indicated by short arrowhead in Fig. 3B). Within the next couple of days, the cells returned to their original morphology and were fusiform or polygonal (long arrowheads indicate fusiform cells and short arrowheads indicate polygonal cells in Fig. 3C). In addition, a typical peak-valley pattern of ASM cells was able to be observed under the optical microscope around 5-7 days following subculture (long arrowheads indicate peaks and short arrowheads indicate valleys in Fig. 3D).
Guinea pig ASM cells identified by immunocytochemistry. Data from immunocytochemical staining with specific $\alpha$-SMA antibodies demonstrated that the cultured cells were guinea pig ASM cells. Immunoreactive products displaying a brown-yellow color located in the cytoplasm were evident. Various shapes in the brown-yellow positive cells, including fusiform, polygonal and irregular triangles were observed under the optical microscope (the longest arrowhead indicates fusiform cells, the long arrowhead indicates polygonal cells and the short arrowhead indicates irregular triangle cells in Fig. 4A). The brown-yellow immunoreactive products were displayed in a skeletal pattern under a high-multiplied microscope (indicated by short arrowheads in Fig. 4B). The immunocytochemical characteristics clearly demonstrated that the cultured cells were typical guinea pig ASM cells.

Growth characteristics of guinea pig ASM cells. As displayed in Fig. 5, guinea pig ASM cells demonstrated a good growth status and the number of cells significantly increased from day 4 to 6 when compared with the previous days. Their doubling time was $3.00 \pm 0.5$ days. The results demonstrated that cells were in a good condition and were able to be employed in the follow-up studies.

Suppressive effect of SPFF on intracellular $\mathrm{Ca}^{2+}$ release. As shown in Fig. 6, the release of intracellular $\mathrm{Ca}^{2+}$ was clearly suppressed by SPFF and the inhibition rate of the drug at 1,0.01 and $0.0001 \mu \mathrm{M}$ was $59.54,55.30$ and $50.46 \%$, respectively. The effect was concentration-dependent when compared with the control. It was clear that SPFF had a better pharmacological activity than mabuterol, the positive drug.

Suppression of $\mathrm{Ca}^{2+}$ release by $S P F F$ is regulated by $I P_{3} R$ and $R y R$ antagonists. As shown in Fig. 7, the release of intracellular calcium was clearly suppressed by $\operatorname{SPFF}(1 \mu \mathrm{M})$ alone, SPFF $(1 \mu \mathrm{M})$ plus 2 -APB (40 $\mu \mathrm{M}$, an $\mathrm{IP}_{3}$ receptor antagonist) as well as SPFF $(1 \mu \mathrm{M})$ plus dantrolene $(40 \mu \mathrm{M}$, a ryanodine receptor antagonist) and the calcium inhibition rate was 56.52, 58.91 and $71.10 \%$, respectively. The effect of SPFF on the $\mathrm{Ca}^{2+}$ levels was almost the same as that in the SPFF plus 2-APB group. However, when SPFF was used in combination with dantrolene, its calcium inhibition rate increased to $71.10 \%$.

\section{Discussion}

Cultured ASM cells are important and useful as an in vitro model for studying the molecular mechanisms underlying either normal or diseased airways. However, it is not easy to obtain in vitro cultured primary guinea pig ASM cells in order to study the pharmacological effects and mechanisms of drugs acting on respiratory diseases. The first reason is the technical obtaining of adequate tissue. Isolating ASM from tissues is difficult. Secondly, it is difficult to remove other cells, including myofibroblasts (1). Therefore, it is important to establish a good method for ASM cell primary culture which is simple, efficient and able to produce repeatable results. The present study described and improved method for the isolation, culture, passage and characterization of guinea pig ASM cells. Trypsin digestion and tissue adherence are two frequently used cultural methods to obtain primary ASM cells. The 

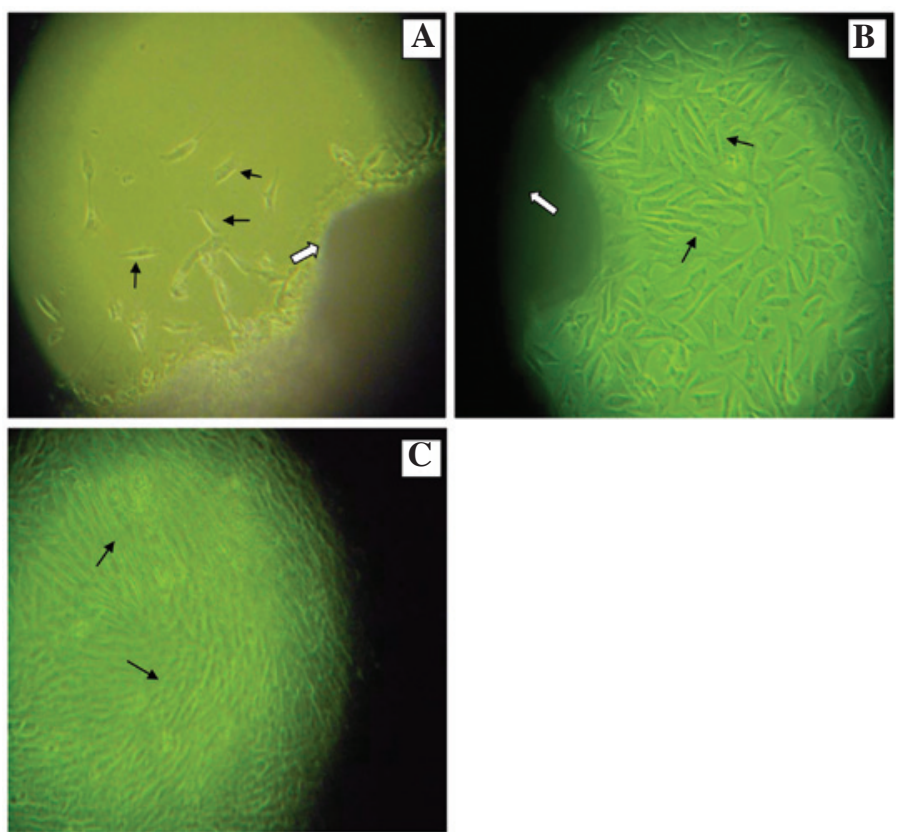

Figure 2. Cultured guinea pig primary ASM cells observed under an optical microscope. (A) GPASM cells cultured for 5-7 days. The cells migrated out from the tissue (indicated by a hollow arrowhead in Figs. 2A and B) and presented all around diffusion growth (indicated by short arrowheads in Fig. 2A), (B) GPASM cells cultured for 8-10 days. The number of cells was significantly increased and fine confluence was observed among adjacent cells (indicated by short arrowheads in Fig. 2B). (C) GPASM cells cultured for 11-14 days. Cells became confluent, interlaced reticulation around cells began to appear and then cells gradually demonstrated numerous branches when the density of cells was highly increased (indicated by short arrowheads in Fig. 2C). ASM, airway smooth muscle; GPASM, guinea pig airway smooth muscle. Magnification, x100.
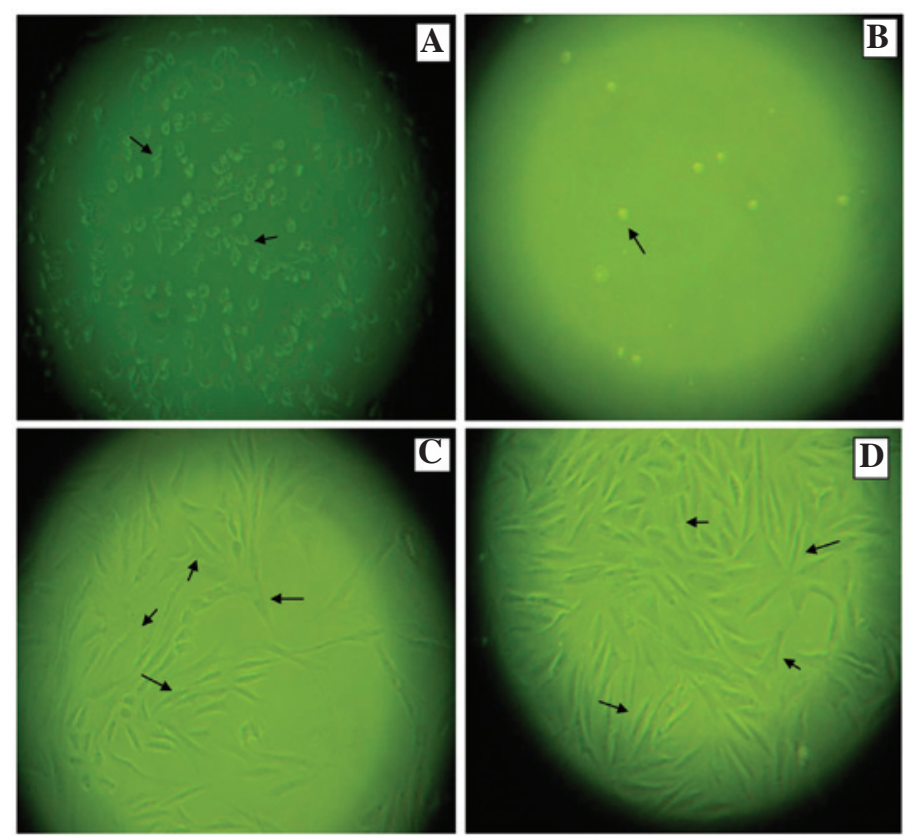

Figure 3. Guinea pig ASM cells following passage were observed under the optical microscope. (A) GPASM cells gradually underwent morphological changes following treatment with $0.25 \%$ trypsin in $0.02 \%$ EDTA, including shrinking into a ball and becoming bright under the optical microscope, which indicated their cytoplasmic rebound (indicated by short arrowheads in Fig. 3A). (B) GPASM cells that were firstly subcultured and then partly transferred into a new cell culture flask became attached to the lining surface of the culture flask $12 \mathrm{~h}$ later (indicated by a short arrowhead in Fig. 3B). (C) GPASM cells returned to their original morphology and were fusiform or polygonal following subculture for 1-2 days (long arrowheads indicate fusiform cells and short arrowheads indicate polygonal cells in Fig. 3C). (D) A typical peak-valley pattern of ASM cells was able to be observed under the optical microscope following subculture for 5-7 days (long arrowheads indicate peak and short arrowheads indicate valley in Fig. 3D). ASM, airway smooth muscle; GPASM, guinea pig airway smooth muscle. Magnification, $\mathrm{x} 100$.

former may readily cause cells a certain amount of damage due to their vulnerable properties and thus, the trypsin digestion time is difficult to control. Consequently, on the basis of present cell culture techniques, a tissue adherence method was applied and the primary ASM cells were successfully cultured via optimizing certain crucial conditions: a) The cell culture 


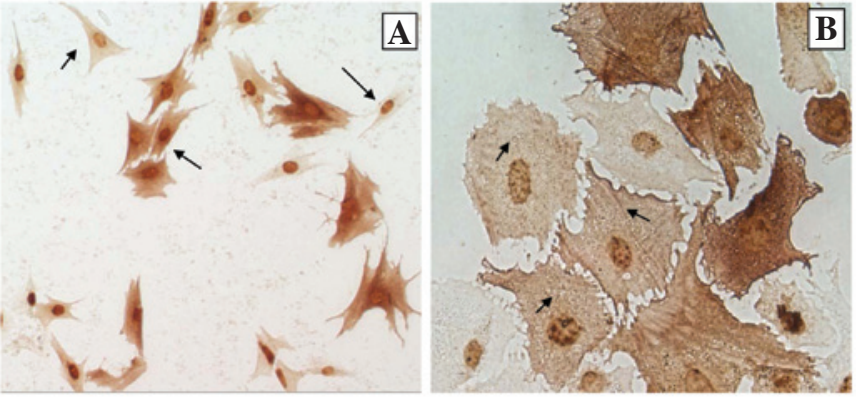

Figure 4. Guinea pig ASM cells under the optical microscope following immunocytochemical identification by $\alpha$-SMA staining. (A) Immunoreactive products located in the cytoplasm displayed a brown-yellow color. Shapes in the brown-yellow positive cells, including fusiform, polygonal and irregular triangles, were observed under the optical microscope (the longest arrowhead indicates fusiform cells, the long arrowhead indicates polygonal cells and the short arrowhead indicates irregular triangle cells in Fig. 4A). (B) The brown-yellow immunoreactive products were displayed in a skeleton pattern under a high-magnification microscope (the short arrowheads indicate a skeleton pattern in Fig. 4B). ASM, airway smooth muscle; $\alpha$-SMA, $\alpha$-smooth muscle actin. Magnification, x400.

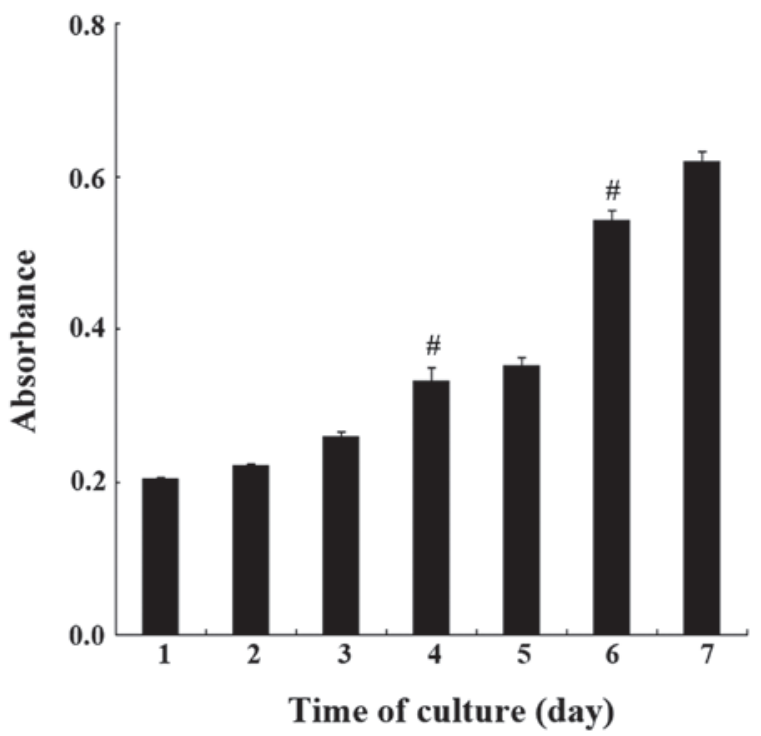

Figure 5. Viability of guinea pig ASM cells cultured in vitro was assessed by MTT assay. Cells were in good condition and the number of the cells significantly increased from day 4 to 6 when compared with cells at the previous days, which is indicated by \#. Data are expressed as the mean \pm standard deviation $(n=5)$. ASM, airway smooth muscle.

flask was preliminarily coated with FBS, which allowed for the tissue masses to easily attach and provided nutritional support for the culture as well; b) the time for turning over the flask was reasonably controlled, which avoided shedding of tissue masses from the cell culture flask; c) connective tissue, bloodstains and tracheal cartilage were removed to the greatest extent, which ensured that a large number of primary ASM cells with high purity were obtained and d) ASM from guinea pigs weighing 150-200 g may provide primary ASM cells with better viability. The whole operation for obtaining guinea pig ASM tissue masses was at $4^{\circ} \mathrm{C}$ and was sterile.

When guinea pig ASM cells migrated out from tissue blocks and grew to two or three generations, there was still a small amount of mixed cells, particularly myofibroblasts, in

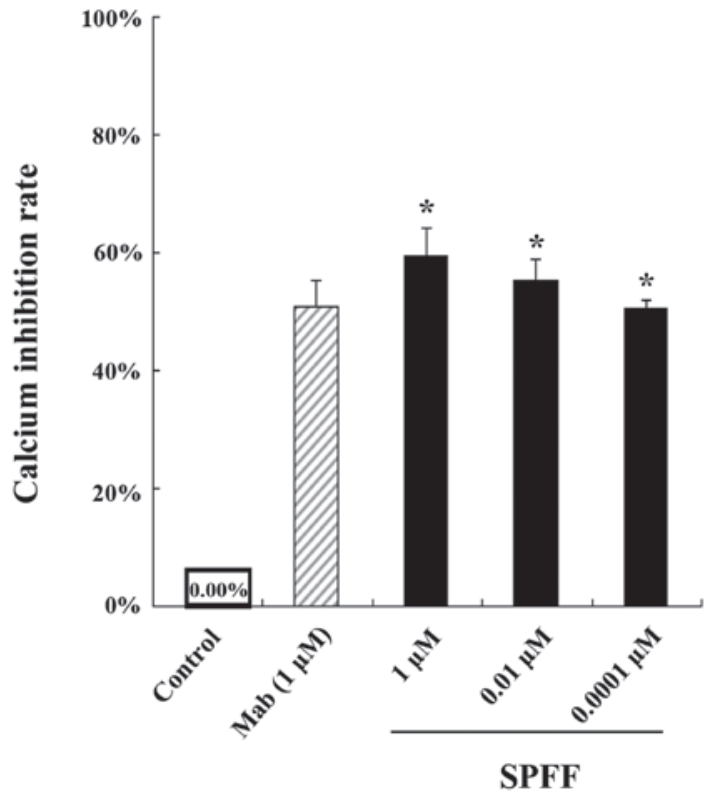

Figure 6. Calcium release inhibition rate of SPFF on the release of intracellular calcium in guinea pig ASM cells. SPFF $(1,0.01$ and $0.0001 \mu \mathrm{M})$ was able to concentration-dependently inhibit the release of intracellular calcium, with inhibition rates of $59.54,55.30$ and $50.46 \%$, respectively. Data are expressed as the mean \pm standard deviation $(n=3)$. ${ }^{*} \mathrm{P}<0.01$ was considered to indicate a statistically significant difference compared with the control. SPFF, 2-(4-amino-3-chloro-5-trifluomethyl-phenyl)-2-tert-butylamino-ethanol hydrochloride; ASM, airway smooth muscle.

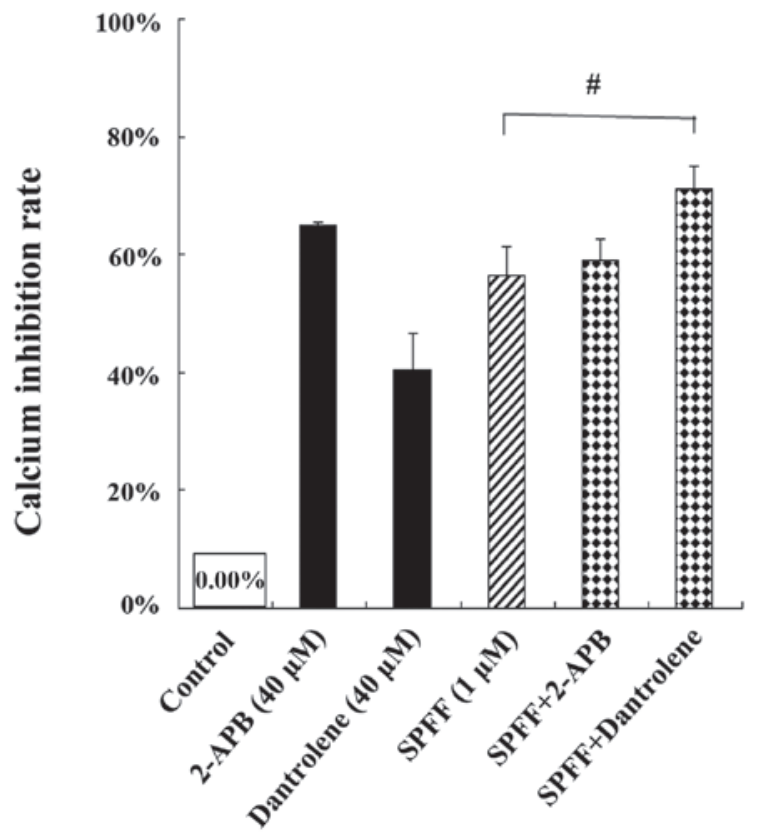

Figure 7. Inhibition of SPFF $(1 \mu \mathrm{M})$ on the release of intracellular calcium was regulated by 2-APB $\left(40 \mu \mathrm{M}\right.$, an $\mathrm{IP}_{3} \mathrm{R}$ antagonist) or dantrolene $(40 \mu \mathrm{M}$, a ryanodine receptor antagonist) in guinea pig ASM cells. As shown, the effect of SPFF on $\mathrm{Ca}^{2+}$ was almost the same as SPFF plus 2-APB, which indicated that the inhibitory effect of the two overlapped; however, when SPFF was combined with dantrolene, its calcium inhibition rate clearly increased. The results implied that the potential mechanism of SPFF on the inhibition of intracellular calcium may be independent of the RyR pathway, but may have a close association with the $\mathrm{IP}_{3} \mathrm{R}$ pathway. Data are expressed as the mean \pm standard deviation $(\mathrm{n}=3) .{ }^{\text {" }} \mathrm{P}<0.05$ was considered to indicate a statistically significant difference when compared with SPFF alone. SPFF, 2-(4-amino-3-chloro-5trifluomethyl-phenyl)-2-tert-butylamino-ethanol hydrochloride; ASM, airway smooth muscle; $\mathrm{IP}_{3} \mathrm{R}$, inositol 1,4,5-trisphosphate receptor; RyR, ryanodine receptor; 1-APB, 2-aminoethyl diphenylborinate. 
the culture. Therefore, the process of subculturing was subsequently used to purify the ASM cells from the mixed culture. ASM cells were isolated and purified with a difference-speed adherence method. Proliferation advantage was also able to be utilized to inhibit the growth of myofibroblasts when there was a large proportion of ASM cells.

A previous study by our group demonstrated that SPFF had a relaxing effect on tracheal smooth muscles. A pharmacodynamic in vivo study of the drug demonstrated an evident increase in lung overflow, lung compliance and cyclic adenosine monophosphate levels in airway tissues when compared with the traditional $\beta 2$-adrenoceptor agonist (9). SPFF also exhibited a new and potential mechanism involving the non-selective potassium channel and the large conductance-activated potassium channel, which differs from traditional $\beta$-adrenoceptor agonists.

The present study further confirmed the effect of SPFF on reducing intracellular calcium release in guinea pig ASM cells cultured by the improved abovementioned method. Furthermore, the potential mechanism of the inhibition of intracellular calcium release by the drug, which ultimately led to the relaxation of tracheal smooth muscles, was researched. The results demonstrated that the effect of SPFF on the release of intracellular calcium was concentration-dependent. Furthermore, the $\mathrm{IP}_{3}$ receptor blocker 2-APB and the ryanodine receptor blocker dantrolene were used to block the two pathways of regulating intracellular calcium release. It was demonstrated that the calcium inhibition rate of SPFF on guinea pig ASM cells was almost the same when incubated with or without 2-APB. However, the calcium inhibition rate of the combined use of SPFF and dantrolene was clearly higher than that of SPFF alone. Accordingly, it was suggested that the inhibitory effects of SPFF and 2-APB were additive and the mechanism of inhibiting intracellular calcium release of the two drugs may be achieved by $\mathrm{IP}_{3} \mathrm{R}$ blocking. By contrast, the inhibitory effect of SPFF and dantrolene may be individual and independent. The present study indicated that the potential mechanism of SPFF on the inhibition of intracellular calcium was independent of the RyR pathway, but may be closely associated with the $\mathrm{IP}_{3} \mathrm{R}$ pathway.

Although the inositol 1,4,5-trisphosphate receptor and the ryanodine receptor are thought to be important in the regulation of intracellular $\mathrm{Ca}^{2+}$ release, the process is intricate and affected by certain cytokines or is closely associated with the cytokines. It has been demonstrated that interleukin (IL)-4 inhibited the magnitude of calcium transients stimulated by ionomycin, which depended on the function of RyR calcium release channels and that the inhibitory effect of IL-4 on calcium transients in ASM cells was able to modulate contraction, proliferation, apoptosis, migration and secretion of mediators $(4,10)$. Furthermore, IL-8 is able to trigger $\mathrm{Ca}^{2+}$-release and ASM cell contraction and migration, which suggests that IL-8 may contribute to airway narrowing, airway hyperresponsiveness and airway remodeling in airway diseases, including asthma and COPD (6). IL-13 is able to augment agonist-induced contraction of the tracheal ring and enhance the frequency of leukotriene D4-induced $\mathrm{Ca}^{2+}$ oscillations and finally increase intracellular $\mathrm{Ca}^{2+}$ concentrations in human ASM cells, which may be co-operatively modulated by the $\mathrm{IP}_{3} \mathrm{R}$ and/or RyR system (11). Consequently, the factors that lead to the fluctuation of the intracellular calcium concentration are numerous and the exact mechanisms by which SPFF, a novel $\beta 2$-adrenoceptor agonist, inhibits the release of intracellular calcium in ASM cells remains to be elucidated. Although the potential mechanisms of SPFF on the inhibition of intracellular calcium are only preliminarily discussed in the present study, it provides useful information for follow-up studies. In future studies, we endeavor to identify new targets upstream or downstream of the $\mathrm{IP}_{3} \mathrm{R}$ signaling pathway and to ultimately elucidate the exact mechanism of the inhibition of intracellular calcium release and relaxation of ASM by SPFF.

In the present study, a method for guinea pig ASM cell culture was successfully established and the purity and viability of ASM cells obtained were better than that those obtained via the traditional method. The drug SPFF, a novel $\beta 2$-adrenoceptor agonist, was able to concentration-dependently decrease $\mathrm{Ca}^{2+}$ levels in the cells and the potential mechanism of the inhibition of intracellular calcium by SPFF may be closely associated with the $\mathrm{IP}_{3} \mathrm{R}$ pathway, which may be conferred as one of the mechanisms of its relaxant effect. However, factors that lead to decreases or fluctuation of intracellular calcium levels and ASM relaxation or contraction are numerous and the exact mechanism by which SPFF inhibits the release of intracellular calcium involving the $\mathrm{IP}_{3} \mathrm{R}$ pathway and eventually relaxes airway smooth muscles remains to be elucidated.

\section{Acknowledgements}

This study was supported by grants from the Education Department of Liaoning (no. L2011169).

\section{References}

1. Camoretti-Mercado B: Targeting the airway smooth muscle for asthma treatment. Transl Res 154: 165-174, 2009.

2. Woodruff PG: Gene expression in asthmatic airway smooth muscle. Proc Am Thorac Soc 5: 113-118, 2008

3. Liu QH, Zheng YM and Wang YX: Two distinct signaling pathways for regulation of spontaneous local $\mathrm{Ca} 2+$ release by phospholipase $\mathrm{C}$ in airway smooth muscle cells. Pflugers Arch 453: 531-541, 2007.

4. Ethier MF and Madison JM: IL-4 inhibits calcium transients in bovine trachealis cells by a ryanodine receptor-dependent mechanism. FASEB J: 20: 154-156, 2006.

5. Pelaia G, Renda T, Gallelli L, et al: Molecular mechanisms underlying airway smooth muscle contraction and proliferation: implications for asthma. Respir Med 102: 1173-1181, 2008.

6. Govindaraju V, Michoud MC, Al-Chalabi M, Ferraro P, Powell WS and Martin JG: Interleukin-8: novel roles in human airway smooth muscle cell contraction and migration. Am J Physiol Cell Physiol 291: C957-C965, 2006.

7. Gan LL, Wang MW, Cheng MS and Pan L: Trachea relaxing effects and beta2-selectivity of SPFF, a newly developed bronchodilating agent, in guinea pigs and rabbits. Biol Pharm Bull 26: 323-328, 2003.

8. Mosmann T: Rapid colorimetric assay for cellular growth and survival: application to proliferation and cytotoxicity assays. J Immunol Methods 65: 55-63, 1983.

9. Hao Z, Zhang Y, Pan L, et al: Comparison of enantiomers of SPFF, a novel beta2-Adrenoceptor agonist, in bronchodilating effect in guinea pigs. Biol Pharm Bull 31: 866-872, 2008.

10. Madison JM and Ethier MF: Interleukin-4 rapidly inhibits calcium transients in response to carbachol in bovine airway smooth muscle cells. Am J Respir Cell Mol Biol 25: 239-244, 2001.

11. Matsumoto H, Hirata Y, Otsuka K, et al: Interleukin-13 enhanced $\mathrm{Ca} 2+$ oscillations in airway smooth muscle cells. Cytokine 57 : 19-24, 2012. 